\title{
Using Quasi-Monoenergetic Photon Sources to Probe Photo-Fission Resonances
}

M. S. Johnson, J. M. Hall, D. P. McNabb, M. Tuffley, M. Ahmed, S. Stave, H. Weller, H. Karwowski, J.

Thompkins

August 13, 2010

CAARI

Ft Worth, TX, United States

August 8, 2010 through August 13, 2010 
This document was prepared as an account of work sponsored by an agency of the United States government. Neither the United States government nor Lawrence Livermore National Security, LLC, nor any of their employees makes any warranty, expressed or implied, or assumes any legal liability or responsibility for the accuracy, completeness, or usefulness of any information, apparatus, product, or process disclosed, or represents that its use would not infringe privately owned rights. Reference herein to any specific commercial product, process, or service by trade name, trademark, manufacturer, or otherwise does not necessarily constitute or imply its endorsement, recommendation, or favoring by the United States government or Lawrence Livermore National Security, LLC. The views and opinions of authors expressed herein do not necessarily state or reflect those of the United States government or Lawrence Livermore National Security, LLC, and shall not be used for advertising or product endorsement purposes. 


\author{
Using Quasi-Monoenergetic Photon Sources to Probe Photo-Fission Resonances \\ Micah S. Johnson ${ }^{a}$, James M. Hall ${ }^{\mathrm{a}}$, Dennis P. McNabb ${ }^{\mathrm{a}}$, Michael J. Tuffley ${ }^{\mathrm{b}}$, \\ Mohammed W. Ahmed ${ }^{c}$, Sean Stave ${ }^{c}$, Henry R. Weller ${ }^{c}$, Hugon Karwowski ${ }^{\text {d }}$, Jeromy \\ Thompkins $^{\mathrm{d}}$ \\ ${ }^{a}$ Lawrence Livermore National Laboratory, Livermore CA 94550 \\ ${ }^{\mathrm{b}}$ San Jose State University, San Jose CA 95192 \\ ${ }^{\mathrm{c}}$ Duke University, Durham NC 27708 \\ ${ }^{\mathrm{d}}$ University of North Carolina, Chapel Hill NC 27599
}

Abstract: We present preliminary results of photo-fission measurements of uranium isotopes with the quasi-monoenergetic gamma-ray source, HIGS. The measurements were performed to search for photo-fission resonances. We discuss potential applications to use photo-fission resonances to identify special nuclear material in cargo containers. We discuss the importance of quasi-monoenergetic gamma-ray sources for this kind of application.

PACS: 24.30.Gd, 25.40.Ny, 25.60.Dz, 25.85.Jg

Keywords: photo-fission, fission, fission neutrons, national security, cross-sections.

Introduction: Quasi-monoenergetic gamma-ray sources are being developed to provide higher fluxes and lower bandwidths. One application of this type of source is to penetrate containers and probe for special nuclear material (SNM). These sources may be gamechangers in the national security sector to detect SNM in various containers. Many efforts are underway to study the efficacy of using these sources with processes such as nuclear resonance fluorescence (NRF) to detect, map, and assay containers for isotopes of interest. LLNL is part of a large collaboration to study the efficacy of NRF for detection and assay [1]. LLNL is also part of a collaboration to look at photo-fission signatures with very intense, low-bandwidth sources to detect SNM. One of the major benefits of using low bandwidth sources is noise reduction.

Actinides have a relatively low fission threshold compared to other nuclei. In fact, very few nuclei have neutron production thresholds (fission neutrons, photodisintegration neutrons, etc...) that are comparable to actinides. Deuterium is an example of a nucleus that has a low neutron production threshold $(2.2 \mathrm{MeV})$. Deuterium is expected to be a large background component for neutron detection with photon sources because of its ubiquity in nature. Quasi-monoenergetic gamma-ray sources can mitigate this background by focusing most of its flux on photo-fission channels. Bremsstrahlung sources integrate the photo-fission channels of the isotope of interest but will also integrate unnecessarily over the photo-neutron production channels of deuterium (and other present isotopes

Photo-fission may offer a fast method to pass or fail a container when looking for fissile material in comparison to other techniques, such as NRF, because the integrated cross section over all beam energies are larger for photo-fission. Furthermore, both neutrons and photons are emitted in photo-fission. The presence of two signatures can be a benefit. Photons are attenuated based on material density and Z. Neutrons, 
however, have different moderation and attenuation characteristics in different materials. Detailed information regarding the energy anisotropy of neutrons from fission resonances using polarized photon sources and correlations with gamma-rays could be used to help reject backgrounds or perhaps even to isotopically identify fissile materials.

Bohr first suggested [2] that near the saddle point of fission, a nucleus will be relatively cold and states at the saddle point should resemble spectra of its normal deformed shape. The incident photon is absorbed, exciting a complicated, normallydeformed state. The excited nucleus has some probability of increasing its deformation and undergoing fission. Near the fission barrier, the possible modes of fission are limited. The deforming nucleus can transition to fission through a lower-energy state near the saddle point and approach the fission barrier with more "kinetic" energy, or can transition through a state of energy similar to the initial state, but with little "kinetic" energy. These states offer a discrete set of resonances near the fission barrier. The search for these resonances is an important aspect of our effort.

Detailed information about the resonances can provide guidance for the bandwidth, energies, and flux requirements for the next generation of quasimonoenergetic gamma-ray sources.

Experimental Method: Photo-fission measurements were performed using the High Intensity Gamma-ray Source (HIGS) at Duke University's Free Electron Laser Laboratory. The energy of the photon source was stepped up in $25 \mathrm{keV}$ steps from 5.0 $\mathrm{MeV}$ to $6.5 \mathrm{MeV}$. The energy range was chosen to look for resonances, which were expected to be near the fission barrier (approx. $5.2 \mathrm{MeV}$ in uranium isotopes). LLNL used ${ }^{238} \mathrm{U}$ as a feasibility measurement to test the method for ${ }^{235} \mathrm{U}$. Thin and thick targets of ${ }^{238} \mathrm{U}$ (in the form of depleted uranium) were used in the feasibility test.

Twelve scintillating neutron detectors were used to measure the neutrons from the fission process. Pulse shape discrimination was used to separate gamma-ray events from neutrons. Time-of-flight was used to determine the neutron energies. The neutron separation energy in ${ }^{238} \mathrm{U}$ is $6.153 \mathrm{MeV}$. This implies that for the energy region that was measured there are no photo-neutrons above $2 \mathrm{MeV}$. The efficiency of the neutron detectors are such that the thresholds are slightly less than $1 \mathrm{MeV}$. Since fission neutrons extend above $2 \mathrm{MeV}$ and the signals below $1 \mathrm{MeV}$ are associated with photo-neutrons or are below thresholds, we have gated on neutrons above $2.0 \mathrm{MeV}$.

The detectors were placed at polar angles of 75-, 90-, and 126-degrees to measure the angular distributions. Three detectors were placed above the beam and three more below the beam, which is the plane perpendicular to the polarization plane. The remainder of the detectors were placed in a similar geometry parallel to the polarization plane (beam-left and beam-right). The placement of the detectors in the different planes were done to measure the anisotropy of neutrons.

The photon flux was measured in-beam with a series of thin plastic paddle scintillators. The paddle detectors were flux-calibrated with a high-efficiency NaI detector and/or an HPGe detector that measured the attenuated flux, on axis, before and after each run.

45 Results and Discussion: The results of the photo-fission measurements of ${ }^{238} \mathrm{U}$ are 46 shown in Figure 1 (only the range from 5.0- to 6.5-MeV are shown in the interest of 
clarity). Our cross-section results were calculated from the measured data using an angle-averaged determination (blue data in figure 1) and from the so-called "A0" coefficient from second-order Legendre fit (red data in figure 1). We have also compared our data to previous measurements by Dickey and Axel [3] and the results are consistent (see figure 1). Deviations from the average cross-sections and the A0 results indicate a lower overlap with the cross-section and $\mathrm{P} 0$ term of the Legendre polynomials. Indeed the A2 coefficients are less than -1.0 in the range from $5.0 \mathrm{MeV}$ to $5.5 \mathrm{MeV}$. The green curve represents the shape of the beam and is superimposed to give the reader a sense of scale.

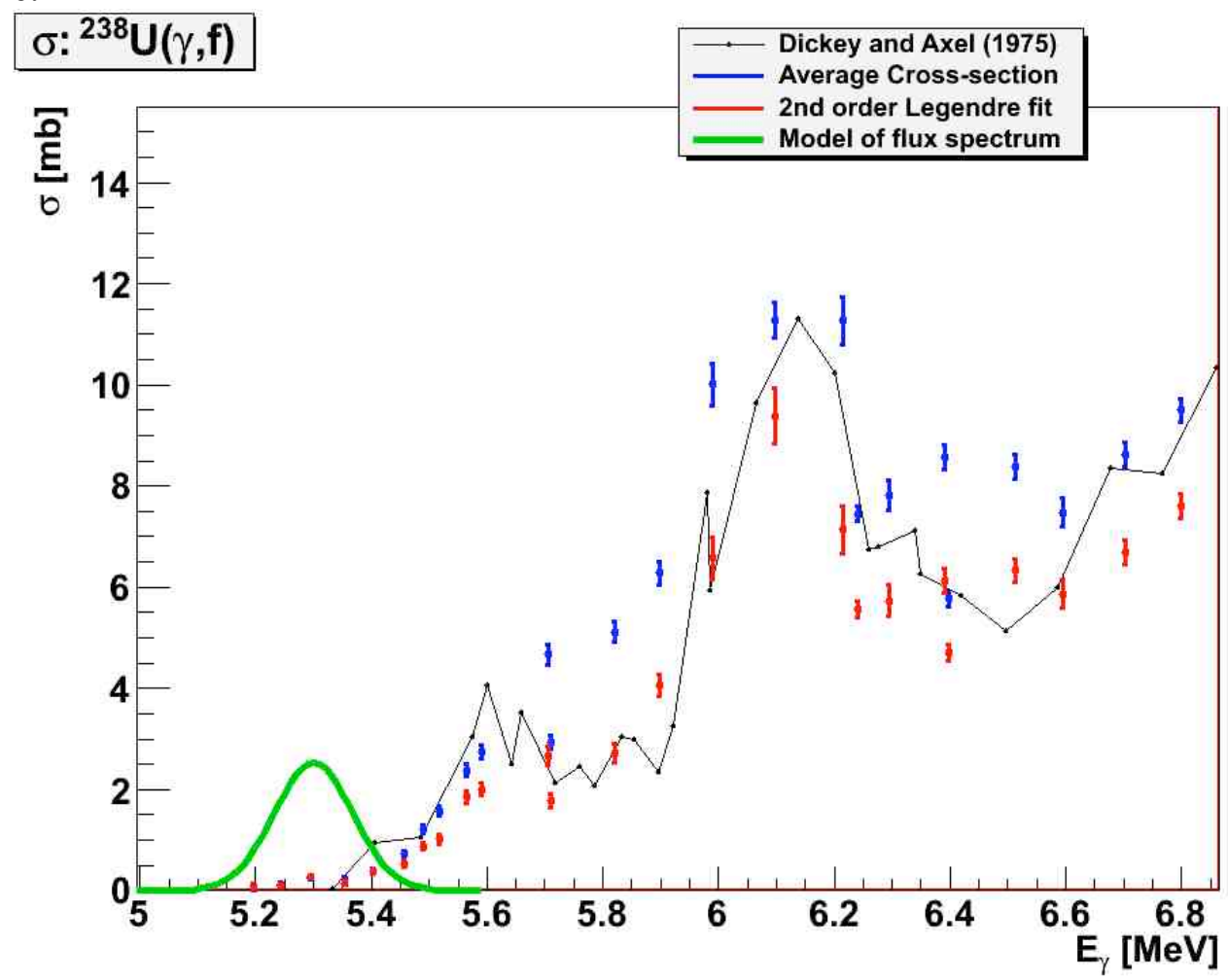

Figure 1: Photo-fission cross-section versus incident photon energy. Blue data represents an average cross-section analysis, and the red data represents a secondorder Legendre fit to the data. The black line represents previous data [3] for comparison. The green curve is a likeness to the beam shape.

It is important to point out that there are two large structures that we have observed and are consistent with Dickey and Axel, centered at 5.6 MeV and 6.2 MeV. These structures are much wider than the beam and could represent cusps or pygmy-like features. From this data it is inconclusive whether or not any resonances exist. Narrow resonances should be at the lowest energies (nearest the barrier). With a broad beam, such as the one from HIGS (approximately $160 \mathrm{keV}$ ) any narrow structures should appear as a hump or shoulder on the overall cross-section. The widths of these shoulders should be no greater than the width of the beam. This is challenging with a broad beam unless the resonance(s) were large. We can use the data for ${ }^{238} \mathrm{U}$ to set a limit on the strength of a single photo-fission resonance at the lowest energy, about $3 \mathrm{MeV}$. Assuming that the green curve is the flux we can integrate the cross-section. We thus set an upper limit on the integrated cross-section at $5.3 \mathrm{MeV}$ to be $95(6) \mathrm{eV}$ barns. This implies that the height, 
$H$, of any hidden resonance is determined simply: $H=3.545 / w$, where, $w$, is the width of the structure, and 3.545 comes from the integrated cross-section.

Recent measurements of ${ }^{235} \mathrm{U}$ (too recent to include here) have shown preliminary indications that there may be underlying structure (resonances) to the above results for ${ }^{238} \mathrm{U}$. The data collected for ${ }^{235} \mathrm{U}$ were taken with $25-\mathrm{keV}$ steps. With careful deconvolution of the data, it may be possible to gain a higher resolution $(25-\mathrm{keV})$ picture of the underlying structures. If the structures are lower in width than what the analysis is capable of extracting, then it is imperative that these structures be re-measured with much narrower beams. This will enable exact determination of the size and shape of the resonance structure (i.e. measure $w$ and $H$ ). Finding the true strength and shape of any proposed resonance will help guide the models for detection protocols with photo-fission probes.

The anisotropy results for ${ }^{238} \mathrm{U}$ show a ratio (perpendicular to parallel) of 2.0(5) at 90 -degrees. This is in contrast to other nuclei including ${ }^{235} \mathrm{U}$, which shows a preliminary result of 1.0(7) at 90-degrees. This may provide an additional signature to detect HEU over natural uranium or DU. The angular distributions show a marked difference, in ${ }^{238} \mathrm{U}$ the $\mathrm{A} 2$ coefficient is 1.3(3) and in ${ }^{235} \mathrm{U}$ the A2 coefficient is $0.03(4)$. This may be a result of the even-even versus even-odd excitations where there are many projections in the odd mass case causing a more isotropic distribution.

The importance of narrow structures for SNM detection is that it allows operators to tune quasi-monoenergetic sources on and off of resonances. By measuring the variations in the emitted photo-neutron count rate, one can determine which isotope (providing no overlaps) is present. Isotopic sensitivity with photo-fission probes would be a game-changer in SNM detection. This is because the integrated cross-section (given above, 95(5) eV barns) is an order of magnitude (or maybe two orders) greater than typical NRF strengths, typically 1 to $10 \mathrm{eV}$ barns. With higher efficiency of detecting neutrons than gamma-rays (from NRF) the counting rates for photo-fission will be much greater (than NRF).

Summary and Future Outlook: We have performed photo-fission measurements of uranium isotopes with a linearly polarized, quasi-monoenergetic gamma-ray source. Our analysis of ${ }^{238} \mathrm{U}$ shows good agreement with prior results. The results indicate narrow structures or the possibility of photo-fission resonances. Data analysis is ongoing and the refined results will be published later for ${ }^{238} \mathrm{U}$ and ${ }^{235} \mathrm{U}$. Detailed information about the resonances can provide guidance for the bandwidth, energies, and flux requirements for the next generation of quasi-monoenergetic gamma-ray sources.

We have also analyzed the data for angular distribution effects and anisotropies in the neutron spectrum and have shown differences between the uranium isotopes. This information may be useful as a signature for detecting SNM in cargo containers.

Acknowledgements: This work performed under the auspices of the U.S. DOE by LLNL under Contract DE-AC52-07NA27344. This effort is support by DHS/DNDO.

\section{References:}

[1] J. Pruet et al., J. Appl. Phys. 99, 123102 (2006)

[2] A. Bohr, Proc. UN Int. Conf. PUAE, 2151 (1956)

[3] P.A. Dickey, and P. Axel, PRL, 35501 (1975) 